Asthma

\section{The continuing challenge to reduce the burden of occupational asthma}

M Sim

What is the best approach?

W ith the reduction in the disease burden from the pneumoconioses in recent years, especially in developed countries, occupational asthma has emerged as the occupational lung disease of greatest importance. It is usually the most common respiratory condition reported in occupational disease surveillance programmes and makes a substantial contribution to the burden of asthma in the community, with an estimated population attributable risk of $15 \%$ and estimated annual cost to the USA of \$US 1.6 billion. ${ }^{1}$ Therefore, the introduction of prevention strategies to reduce the impact of occupational asthma, should be strongly supported.

The editorial by Snashall in this issue ${ }^{2}$ describes the latest proposal by the Health and Safety Commission (HSC) to reduce the incidence of occupational asthma in the United Kingdom. ${ }^{3}$ This article acknowledges the limited success of past HSC prevention programmes. The proposed Strategy has five components corresponding to the key programmes in Securing health together, the HSC's 10 year occupational health strategy, released in 2000. These components include the introduction of an Approved Code of Practice (ACoP), more targeted enforcement, better management systems, improved education, and more research. The primary stated aim of the new strategy is to reduce the incidence of occupational asthma, but in fact many of the proposed actions are appropriately aimed at other aspects of disease burden reduction, such as early detection and more effective case management, not just measures to reduce incidence.

Snashall's article indicates that the Strategy will also include action on asthma made worse by work; an inclusion made after a public consultation phase. While this is undoubtedly a worthwhile aim in terms of good occupational health practice, it is debatable whether it should be part of this particular Strategy. The aggravation of pre-existing asthma is usually due to non-specific triggers, rather than asthmagenic agents, so preventive measures will need to be different from those used to control asthmagens and will apply to a greater number of workplaces. Also, unlike occupational asthma, we have no surveillance data for aggravated asthma, which will make it difficult to monitor the success of the Strategy in the prevention of this problem. Finally, including action on asthma aggravation in the Strategy may dilute the prevention effort which can be directed towards reducing the impact of true occupational asthma.

The target set for occupational asthma reduction in the Strategy is $30 \%$, which is considerably higher than the HSC's overall $20 \%$ reduction target for occupational disease by 2010 . While this higher target is a laudable aim, it will require considerably more resources than those required to achieve a $20 \%$ reduction. A major problems with such targets is that the surveillance data on which they rely can take many years to show the effectiveness of prevention measures. ${ }^{4}$ As the usual period between exposure and disease for occupational asthma is quite short, this should be less of a problem for occupational asthma than for many other occupational diseases of longer latency. One note of caution relates to the proposal in the Strategy to extend and improve SWORD. It will be important to ensure that such changes do not, in themselves, lead to changes in the incidence data over time, for example by changes in case ascertainment, definitions, or reporting processes. ${ }^{4}$ Any future trends in the SWORD data should reflect real changes in the occurrence of occupational asthma in UK workplaces. Other measures will need to be developed to evaluate improvements in early detection and management of cases.

\section{"Much of the detail is yet to be developed"}

Snashall acknowledges that this will be an ambitious programme and that much of the detail of the wide range of proposed preventive measures is yet to be developed. Some of the proposed actions have clear merit, such as the introduction of an ACoP, which was deferred in 1992 and it could be argued should have been introduced earlier. However, other proposed actions may be less effective. One example is the proposal to train general practice nurses to ask some screening questions of all adult asthma clinic attendees. Many of these nurses may see very few, if any, potential occupational asthma cases per year. More peripheral proposals such as this will need careful consideration of the required resources versus the likely benefit in achieving the aims of the Strategy.

One of the debates in public health circles has been whether the thrust of prevention should be directed across the whole population or confined to high risk groups, to make the most efficient uses of scarce resources. The late Geoffrey Rose was a strong advocate of the former approach. ${ }^{5}$ The Strategy is advocating the latter approach, by targeting for prevention the eight asthmagens known to cause about half of the reported cases of asthma in the UK. ${ }^{1}$ Most of these eight asthmagens have been well recognised for many years as causes of occupational asthma and many, such as wood dust, flour, and laboratory animals, cannot be eliminated or substituted. In addition, this targeted approach does not appear to consider the size of the population at risk. It could be argued that the most efficient use of resources is to target those asthmagens where the proportion of cases, in relation to the working population at risk, is highest.

Targeting these more established causes of occupational asthma, where there is a higher knowledge base among notifying physicians, industry, and employees, may repeat the past mistake of "preaching to the converted", which Snashall emphasises as a major factor in lack of success in the past. Occupational asthma cases exposed to less well known asthmagens may be less likely to end up in the SWORD data, but these may be easier to eliminate or substitute, and perhaps it is these types of workplaces where attention should also be directed. Another major identified problem with previous programmes is that they did not reach the small to medium sized enterprises where exposure to asthmagens occurs. It is not clear in the Strategy what the particular approaches will be to reach these types of enterprises this time. Finally, the success rate in asthma case reduction for these top eight targeted asthmagens will need to be higher than $30 \%$, to achieve the overall reduction target of $30 \%$ for all asthmagens.

While the Strategy is a good start, considerable work still remains to be undertaken to develop specific cost-effective 
actions, implement them, and undertake evaluation to determine whether they have achieved a substantial reduction in the occupational asthma disease burden in the UK. It is to be hoped that the HSC can introduce an effective prevention system, which can be used as a model for other countries to follow, and that the proposed Strategy will not follow the same ineffective path as its predecessors.

Occup Environ Med 2003;60:713-714

\section{Author's affiliation}

M Sim, Department of Epidemiology \&

Preventive Medicine, Monash

University, Alfred Hospital,

Commercial Road, Melbourne, Victoria 3004,

Australia

Correspondence to: A/Prof. M Sim,

Department of Epidemiology \& Preventive

Medicine, Monash University,

Alfred Hospital, Commercial Road, Melbourne, Victoria 3004, Australia;

Malcolm.sim@med.monash.edu.au

\section{REFERENCES}

1 Leigh JP, Romano PS, Schenker MB, et al. Costs of occupational COPD and asthma. Chest 2002;121:264-72.

2 Snashall D. Occupational asthma: another fresh start in the UK. Occup Environ Med 2003:60:711-12.

3 HSE. Securing health together - a long term health strategy for England, Scotland and Wales (MISC225). London: HSE Books, 2000

4 Coggon D. Monitoring trends in occupational illness. Occup Environ Med 2001:58:691-3.

5 Hofman A, Vandenbroucke. Geoffrey Rose's big idea-changing the population distribution of a risk factor is better than targeting people at high risk. BMJ 1992;305:1519-20.

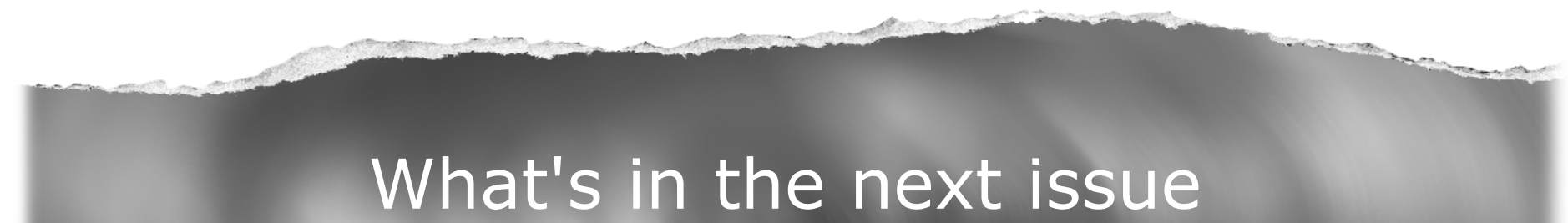

\section{Future content}

See which articles have just been accepted for publication and preview the table of contents for the next issue a month before it is published

\section{www.occenvmed.com}

